\title{
La incidencia de la asimetría en la práctica profesional de la interpretación simultánea árabe-español
}

\author{
Bachir Mahyub Rayaa - Universidad de Granada (D) 0000-0001-5400-5374
}

Recepción: 22.10.2020 | Aceptado: 02.02.2021

Correspondencia a través de ORCID: Bachir Mahyub Rayaa

iD 0000-0001-5400-5374

Citar: Mahyub Rayaa, B. (2021). La incidencia de la asimetría en la práctica profesional de la interpretación simultánea árabe-español. REIDOCREA, 10(2), 1-12.

\begin{abstract}
Resumen: En la interpretación de lenguas existe un debate acerca de si su ejercicio consiste en un proceso cognitivo inalterable e independiente del par de lenguas en el que se trabaja, o si las peculiaridades de determinadas combinaciones lingüísticas podrían alterar ese proceso, lo que variaría de estrategias cognitivas empleadas y recomendaría el abordaje de aspectos formales de las lenguas en la formación de intérpretes. La interpretación simultánea árabe-español (IS AR-ES), en tanto que implica dos lenguas asimétricas lingüística y socioculturalmente, debe abordar este debate y aportar conclusiones empíricas para guiar la docencia y la práctica profesional. Método: Estudio basado en encuesta acerca de la práctica de la IS AR-ES, sus especificidades y la posible incidencia de la asimetría en este par lenguas. Se suministró un cuestionario de 45 preguntas a 12 intérpretes de conferencias AR-ES, casi la totalidad de profesionales que ejercen en España. Resultados: Revelan una notable incidencia de peculiaridades del árabe en la práctica de la IS AR-ES (diglosia, alternancia de código, variación diatópica y diastrática, etc.). Conclusiones: La asimetría en la combinación AR-ES abarca los planos lingüístico y cultural, que se entremezclan, y es más acusada que en las combinaciones español-inglés y español-francés ejercidas por estos intérpretes.
\end{abstract}

Palabras clave: Interpretación

The incidence of asymmetry in the Arabic-Spanish simultaneous interpretation professional practice

Abstract: In language interpretation there is a debate about whether its practice consists of an unalterable cognitive process, independent of the linguistic combination in which one interprets, or the specificities of certain linguistic combinations could affect this process, which would vary from cognitive strategies employed and would recommend addressing formal aspects of languages in interpreters' training. ArabicSpanish Simultaneous Interpreting (AR-SP SI), as it involves two linguistically and socio-culturally asymmetric languages, should address this debate and provide empirical conclusions to guide teaching and professional practice. Method: A survey-based study is conducted on the professional practice of AR-SP $\mathrm{SI}$, its specificities and the possible incidence of asymmetry in this pair of languages. A 45-questions questionnaire was provided to 12 AR-SP conference interpreters, almost all professionals practicing regularly in Spain. Results: They reveal a significant incidence of specificities of the Arabic language during the practice of AR-SP SI (diglossia, code-switching, diatopic and diastratic variation, etc.). Conclusions: The asymmetry in the AR-SP combination encompasses the linguistic and cultural frames, which intermingle, and it seems more pronounced than in the English-Spanish and French-Spanish pairs covered by the interpreters that participated in the study.

Keywords: Interpreting

\section{Introducción}

Para la Teoría del Sentido (Seleskovitch y Lederer, 1984: 108; Déjean Le Féal, 1998: 43, entre otros), la interpretación es un acto cognitivo independiente de la combinación lingüística. No obstante, la literatura previa subraya desde finales de los 70 que determinados pares de lenguas presentan dificultades formales diferentes (Longley, 1978 y Wilss, 1978) y exigen estrategias específicas (Stenzl, 1989: 24). A este respecto, se registran trabajos que recomiendan la necesidad de que la didáctica de la interpretación tenga en cuenta las especificidades de la combinación lingüística, especialmente en aquellos pares que presentan más dificultades de tipo formal y sintáctico o, dicho de otro modo, que presenten mayor asimetría (llg 1978 y Gile 1995). Kelly (2003), por su parte, señala, desde la perspectiva de la traducción, que a pesar de 
las similitudes observables en toda actividad profesional, existen diferencias regionales y nacionales, culturales y profesionales que deben ser tenidas en cuenta a la hora de diseñar los planes curriculares. El problema teórico planteado tiene, pues, repercusiones profesionales y docentes.

En lo que respecta a la investigación en interpretación de conferencias el par de lenguas árabe-español, se observa que ha sido muy escasa hasta bien entrada la segunda década del siglo XXI (Mahyub Rayaa, 2015). Esto, sin embargo, se contrapone a su notable demanda profesional y su plena inclusión en los planes de formación universitarios españoles. Por ello, la investigación en interpretación de/hacia el árabe todavía no ha aportado conclusiones fundadas que ayuden a resolver el problema teórico arriba planteado, si bien en un estudio reciente se responde a algunos de los interrogantes relacionados con esta cuestión (Mahyub Rayaa, 2020). A este trabajo habría que sumar el de Hassan (2009), en el que se aborda la cuestión que aquí se plantea: si debemos seguir concibiendo el ejercicio de la interpretación como un proceso cognitivo inalterable o si, por el contrario, las especificidades de determinados pares de lenguas pueden incidir y, por ende alterar, en ese proceso cognitivo. Hassan (2009) critica, en este contexto, la Teoría del Sentido y afirma que la especificidad del par de lenguas incide notablemente en la práctica, lo que debe incorporarse al ámbito de la formación. En su análisis, de carácter intuitivo, acerca de la interpretación simultánea árabe-francés, la autora destaca, entre otras especificidades, la diferencia entre las estructuras lingüísticas y el orden de los sintagmas -lo que más arriba se indicaba como aspectos formales y sintácticos (Ilg 1978 y Gile 1995)-, y propone examinar en el futuro la incidencia de la variación en árabe, los problemas de terminología y las diferencias culturales.

Precisamente los problemas derivados de la variación diatópica y diastrática en la lengua árabe han sido objeto de un gran interés en los estudios de filología árabe desde la segunda mitad del siglo XX (Ferguson 1959; Badawi 1973; Corriente 1977; Youssi 1983; Versteegh 1997, y Ferrando 2001, entre otros). El impacto de la variación en el ámbito de la interpretación del árabe, subrayado por Hassan (2009), no ha sido investigado empíricamente hasta el momento, y se ha abordado considerándolo un fenómeno casi exclusivo de la interpretación para los servicios públicos (Feria 2001, Taibi 2007 y Ortega Herráez 2007), ya que supuestamente en la modalidad de interpretación simultánea el árabe moderno estándar es el único registro al que debe verter el intérprete y, teóricamente, el único utilizado por los oradores en contextos formales. Esta regla no escrita ya ha sido rebatida por la literatura previa (Wilmsen, 2003; Mahyub Rayaa, 2013, 2015 y 2020), que ha constatado que, con cierta frecuencia, el orador árabe cambia a lo largo de su disertación del árabe estándar al árabe dialectal.

A pesar de lo dicho, la investigación previa sigue sin zanjar el problema teórico planteado y, en lo que se refiere a la interpretación AR-ES, aún queda por averiguar el impacto real y las consecuencias de la asimetría en esta combinación sobre la práctica de la interpretación simultánea (IS) entre las dos lenguas.

\section{Hipótesis y objetivos}

El presente trabajo parte de la hipótesis de que la asimetría lingüística y sociocultural a prioripatente en la combinación lingüística árabe-español incide en el proceso cognitivo de la IS entre estas dos lenguas, a diferencia de otras combinaciones lingüísticas más simétricas (francés-español, italiano-español, etc.). Esta hipótesis se sustenta en la literatura previa antes descrita y se apoya en las conclusiones que apuntan a la influencia de aspectos formales de pares de lenguas específicos y al uso de estrategias 
diferentes en el ejercicio la IS, así como apuntan a la recomendación de abordar sus peculiaridades con ejercicios específicos durante la formación de intérpretes.

El trabajo pretende, por lo tanto, averiguar qué influencia tiene la asimetría percibida de la combinación lingüística árabe-español en el proceso cognitivo durante la práctica de la IS y cómo se manifiesta en caso de existir tal influencia. Con ello, se quiere explorar la posible influencia de las peculiaridades del par de lenguas AR-ES en el ejercicio de la IS, así como analizar las posibles estrategias que emplean los intérpretes profesionales para superarlas y/o neutralizar dichas peculiaridades.

\section{Material y métodos}

Este estudio se basa en un cuestionario que sondea la percepción de los profesionales del sector. Tras una recopilación previa se definió un grupo compuesto por 16 intérpretes de conferencias árabe-español que representan la práctica totalidad de los profesionales que se dedican habitualmente a la IS AR-ES en España. A todos ellos se les envió un cuestionario por vía telemática. Se recibieron 12 cuestionarios cumplimentados anónimamente, lo que supone una tasa de respuesta del $75 \%$ (porcentaje de error del $13 \%$ y nivel de confianza del $90 \%$ ).

Para la elaboración del cuestionario se partió de uno previo empleado en el marco de la tesis doctoral de Mahyub Rayaa (2015), que a su vez bebe de otras encuestas empleadas por Iglesias Fernández (2003) y Manuel Jerez (2006). El cuestionario incluyó 45 preguntas (14 preguntas cerradas, 20 abiertas, y 11 semicerradas) divididos en tres apartados: perfil de los sujetos, docencia y práctica profesional, y empleo de materiales didácticos. Para el presente trabajo nos hemos limitado a analizar los datos de aquellas peguntas relacionadas con la asimetría en la práctica profesional o sus implicaciones en la docencia.

\section{Resultados}

\section{Perfil de los encuestados}

La población estuvo compuesta por 10 hombres (83\%) y 2 mujeres (17\%), con edades de entre 25 y 60 años; el $75 \%$ tenían entre 35 y 50 años. Su experiencia profesional oscilaba entre 10 y más de 30 años, y el $66 \%$ atesoraba de 15 a 20 años de experiencia profesional en IS AR-ES.

El $67 \%$ de los encuestados eran titulados superiores. El $42 \%$ cursaron formación reglada en Traducción e Interpretación, el $25 \%$ otro tipo de estudios superiores (Filología Semítica, Filología Hispánica y Ciencias Políticas) y el 33\% NS/NC. Independientemente de los estudios superiores realizados, el $75 \%$ recibió alguna formación en IS antes de ejercerla profesionalmente. El periodo de formación en interpretación oscila entre 6 meses y 4 años.

La lengua materna del $83 \%$ es el árabe y el español, su principal lengua extrajera. El $92 \%$ afirma que interpreta en los dos sentido, directa e inversa árabe-español. Para el $50 \%$ el francés es segunda lengua extranjera, y para el $42 \%$ es el inglés. Todos afirman que sus segundas lenguas extranjeras son pasivas, esto es, que interpretan de ellas al árabe, pero no a la inversa. 
El $100 \%$ sostiene que realiza otras labores además de la interpretación. El $75 \%$ se dedica a la traducción escrita. El 50\% son docentes de traducción y/o de interpretación de forma esporádica o habitual. El 58\% son intérpretes autónomos (trabajadores por cuenta propia); el resto son trabajadores por cuenta ajena.

Se trata, por lo tanto, de un colectivo muy experimentado, con un nivel de formación medio-alto, lo que hace de su percepción un elemento muy significativo para propósito de este estudio.

\section{Especificidades de la interpretación simultánea árabe-español}

El 100\% de los sujetos afirma que la combinación lingüística árabe-español presenta especificidades propias. En caso de respuesta afirmativa se solicitó seleccionar una o varias de las opciones que se les ofrecían para describir la naturaleza de esas especificidades: sintaxis y estructura de las oraciones, estilos, registro, estructura discursiva, factores socioculturales y otros. El 75\% consideró que factores formales (sintaxis y estructura de la oración) y culturales son específicos de la combinación; casi el $60 \%$ afirmó lo propio del registro y la estructura discursiva, y el $50 \%$ de los estilos lingüísticos.

En el apartado "otros" se ofrecía la posibilidad de especificar, y dos sujetos lo hicieron. El primero resaltó el problema de la diglosia y de la variación en el uso entre árabe dialectal y el árabe moderno estándar o fusḥa, con esto se apunta a que los oradores utilizan el árabe informal incluso en contextos formales como las conferencias. El segundo subrayó los fenómenos de code-switching o alternancia entre lenguas: los oradores, afirmó, pasan del árabe al francés o inglés y repentinamente vuelven al árabe.

\section{Dificultades adicionales, estrategias y soluciones}

El $84 \%$ de los sujetos afirmó que esas especificidades suponen una dificultad adicional para el intérprete en comparación con otras combinaciones lingüísticas que también ejercen profesionalmente, el $8 \%$ lo niega y el $8 \%$ restante NS/NC. En caso de respuesta afirmativa se solicitaba en campo abierto una explicación sobre cómo solucionar el problema o qué estrategias se aplican para superar dicha dificultad. Las soluciones y estrategias apuntadas son las siguientes:

- Al interpretar del árabe al español:

- Traducir el sentido y no atender a la forma.

- Ralentizar la interpretación, sintetizar o parafrasear, en particular cuando el ordador utiliza una variedad dialectal difícil para el intérprete.

- Ante citas literales de un registro lingüístico muy alto o de una variedad arcaica de la lengua árabe, en lugar de traducir, aclarar lo que hace el orador o parafrasear en estilo indirecto el tenor de la cita que haya aportado.

- En caso de alternancia de códigos [code-switching] que suponga una dificultad para el intérprete, ya sea entre variedades de la lengua árabe o entre el árabe y otra lengua, recurrir al compañero de cabina u omitir lo que no se haya entendido.

\section{Enfoques didácticos específicos}

Antes de entrar en los resultados recopilados en este epígrafe, conviene recordar que el $50 \%$ de los encuestados son docentes de traducción y/o de interpretación, lo que imprime una cierta relevancia a sus respuestas en este campo. Ante la pregunta de si deben aplicarse enfoques didácticos específicos para combinaciones lingüísticas 
concretas, el $50 \%$ de los encuestados respondieron que sí se debería, el $25 \%$ opinó que no y el $25 \%$ NS/NC. Curiosamente, todos los encuestados que abogan por introducir un planteamiento didáctico específico para árabe-español se han formado en interpretación y ejercen la docencia de la traducción y la interpretación árabe-español, mientras que los que se manifiestan en contra son aquellos que indicaron tener una formación autodidacta.

Si la respuesta era afirmativa se solicitaba en campo abierto una explicación adicional. Las principales respuestas fueron:

- Se debe potenciar el dominio de la síntesis para salvar las diferencias discursivas entre el árabe y español (ya que el árabe es mucho más prolijo) y así se aprende a ganar tiempo.

- Potenciar las herramientas de adaptación de las estructuras sintácticas de una lengua a otra, con automatismos, y de formulación de los numerales.

- Potenciar los conocimientos culturales para resolver los problemas derivados de interpretar registros muy elevados (Corán y poesía) o referencias históricas.

\section{Aspectos complementarios}

A continuación, figuran las observaciones formuladas en el apartado final de respuesta libre. Se recogen aquí aquellas relacionadas con el tema de este trabajo:

- La combinación AR-ES exige, de forma específica, un nivel cultural más elevado.

- También, por la riqueza de registros y variedades de la lengua árabe, requiere un dominio lingüístico mayor.

- Una mayor capacidad de adaptación, ya que la combinación AR-ES presenta una evolución más rápida que otras con la creación de nuevos términos y vocabulario.

- Lo anterior exige, entre otras cosas, potenciar la especialización temática durante el período de formación.

- En la combinación AR-ES es preciso revalorizar el período de formación, reforzar los conocimientos de teoría de la interpretación en todas sus modalidades, abandonar la idea de que el intérprete nace y no se hace, y potenciar la toma de conciencia de los aspectos ético-profesionales.

\section{Discusión de los resultados}

\section{Distancia lingüística}

Obviamente, la asimetría de tipo formal a la que aluden los encuestados puede explicarse por la distancia lingüística, variable difícil de medir y para la que, hasta donde nos alcanza, no existen datos empíricos en relación con el binomio árabe-español (Borin y Saxena 2013). En cualquier caso aquí no nos interesa la distancia lingüística en sí misma, sino sus consecuencias. Beenstock et al. (2001) y Chiswick y Miller (2005), entre otros, han abordado esas consecuencias con datos relativos a la competencia lingüística entre el hebreo y el inglés entre inmigrantes, respectivamente, en Israel y en EEUU y Canadá. No olvidemos que el árabe y el hebreo pertenecen a la misma familia (las lenguas semíticas). Los resultados podrían apuntar esclarecedoramente a las consecuencias de la distancia entre el árabe y el inglés, frente a la distancia entre el árabe y el hebreo: los inmigrantes en Israel con mayor competencia en hebreo son los arabófonos, mientras que solo los inmigrantes japoneses, coreanos y cantoneses en 
EEUU presentan una competencia en inglés menor a la de los árabes, a los que solo igualan los inmigrantes provenientes de Laos, los hablantes de mandarín y los vietnamitas.

\section{Distancia cultural}

La asimentria cultural y su influencia en el proceso de la traducción (Hatim 1997) y de la interpretación (Al-Salman y Al-Khanji 2002: 624; Hassan 2009, Mahyub Rayaa y Zarrouk 2013 , entre otros) ya ha sido puesta de manifiesto por la literatura especializada. Los encuestados, y he aquí lo revelador de su experiencia, insisten en que las asimetrías lingüísticas y culturales se entremezclan, puesto que las segundas terminan reflejándose en el plano formal. Los sujetos insisten especialmente en las dificultades derivadas de la terminología religioso-jurídica islámica, no pocas veces carente de equivalente en español.

Esta asimetría cultural tiene mayor incidencia y queda más patente en otra de las modalidades de la interpretación: la interpretación para los servicios públicos. Autores que estudian este ámbito (Herrero 1995, Feria 1999 y Ortega Herráez 2010) sitúan la asimetría cultural como una de las cuestiones más importantes en el perfil del intérprete que vaya ejercer en esta modalidad, ya que tendrá que adaptar los registros, los conceptos especializados, la terminología, aclarar diferencias culturales que puedan incurrir en malentendidos, intervenir a iniciativa propia, etc.

\section{Alternancia de código y variación de registro}

Con independencia de la distancia lingüística entre el árabe y el español, los encuestados subrayan la dificultad que supone una realidad lingüística árabe diglósica, multiglósica o pluriglósica (Youssi 1983, Ferrando 2001: 135, 184, y Lamrani 2002, entre otros). La literatura subraya que, aunque la diglosia "no es en sí misma nada excepcional, sí resulta llamativo el hecho de que la distancia entre los dos registros extremos [en árabe] es ciertamente superior a la habitual en las lenguas de nuestro entorno" (Ferrando 2001: 136). Esta situación, como hemos podido comprobar, supone un gran problema incluso para intérpretes con dilatada experiencia y cuya lengua materna es el árabe.

Aunque la alternancia entre distintas variedades de la lengua árabe es mayor cuanto más espontáneo sea el discurso, incluso en discursos informales puede el orador recurrir a ella para, entre otras cosas, explicar un concepto oscuro en registro formal, infundir naturalidad y empatizar con el público, introducir un tono humorístico o irónico, recurrir a la intertextualidad o jugar con diferentes sentidos de una palabra o expresión en diferentes registros (Holes 1993: 13-45, Bassiouney 2006 y Moshref 2012). En cualquier caso, incluso utilizando un registro formal, la variación diafásica se hará patente, en particular, en el acento, aspecto en el que encontramos similitudes con la situación en combinaciones que incluyen el francés y el inglés.

Aunque los encuestados no lo mencionen, no todo es negativo para el intérprete en esta realidad multiglósica, siempre que conozca la variedad dialectal utilizada por el orador (Mahyub Rayaa, 2020). Por una parte, el discurso espontáneo dialectal es generalmente menos denso y presenta más redundancias lingüísticas que un discurso en árabe estándar, especialmente que uno leído en este registro formal; por la otra, al no ser el árabe estándar la lengua de expresión natural de ningún orador, la velocidad de dicción en ese registro será menor cuando se trate de un discurso espontáneo y la presión para el intérprete, también. No obstante, esta ventaja se convierte en un hándicap cuando el 
orador lee un discurso escrito en árabe estándar, dada la densidad de este registro y la elevada velocidad de lectura de muchos oradores.

En cualquier caso, los encuestados presuponen en sus respuestas que la variación solo es posible para el resto de los intervinientes en el acto comunicativo, nunca para el intérprete simultáneo, que está obligado a usar el registro árabe moderno estándar por la formalidad del acto. La situación, debemos advertir, se invierte en la interpretación consecutiva y bilateral para los servicios públicos o las autoridades policiales y judiciales, en la que el intérprete suele emplear el árabe informal, salvo que el cliente solicite otra cosa (Feria 2001, Taibi 2007, Ortega Herráez 2007 y Mahyub Rayaa, 2015). Como consecuencia, en la práctica, la contratación del equipo de intérpretes debe considerar el perfil lingüístico de los oradores o clientes árabes y sus preferencias.

Por otro lado, los encuestados subrayan la dificultad que supone la variación terminológica, que en árabe se manifiesta tanto en términos que aluden a diferentes conceptos como a conceptos denominados de muy diferentes formas en diferentes países. Se trata de una cuestión ya abordada, entre otros, por Aamami (1997) en relación con la pesca, y por Wilmsen (2003: 77) y Wilmsen y Osama Youssef (2009: 191-210), en relación con la agricultura y la alimentación. En este último trabajo se subraya que, si bien el fenómeno existe en otras lenguas, incluido el inglés, la variación terminológica afecta en árabe a todas las disciplinas científicas y tecnológicas (Wilmsen y Osama Youssef 2009: 205-207).

Las raíces de esta variación datan de mediados del siglo XIX, cuando en Oriente Próximo y Egipto comienza la modernización de la lengua árabe y el acuñado de la terminología científica y tecnológica moderna (Abdul Aziz, 2003: 9-28 y Crozet 2003: 927). La situación se agravó a lo largo del siglo XX bajo la influencia de las lenguas coloniales y, aunque en las últimas décadas se ha procurado normalizar la terminología y los neologismos (Jordan Academy of Arabic 2012-2015', por ejemplo), nada indica que el problema pueda resolverse a corto plazo.

Esta variación discurre en paralelo con el uso combinado del término árabe y su equivalente en una lengua extranjera, hecho al que alude uno de los encuestados. Este tipo de code-switching se observa tanto en la variedad formal como en la informal y permite al orador, a menudo con formación especializada en inglés o francés, salvar la incertidumbre generada por la variación terminológica en árabe. Este fenómeno ha sido descrito por Abulghar (2003: 46-53) para el ámbito de la medicina y se documenta en discursos reales que fueron interpretados del árabe al español en Mahyub Rayaa y Zarrouk (2013: 23-43).

\section{Soluciones indicadas para la asimetría}

Las estrategias a las que apuntan los encuestados (véase en "Resultados") coinciden con algunas de las descritas por Al-Salman y Al-Khanji (2002: 611-612) para la combinación AR-IN según el testimonio de intérpretes con al menos cinco años de experiencia encuestado por los dos autores. En su caso enumeran las siguientes estrategias:

1. "Saltos" (skipping): omisión de redundancias y repeticiones innecesarias. Es a lo que apuntan los intérpretes árabe-español encuestados en nuestro trabajo al pedir que se potencie la habilidad de síntesis.

\footnotetext{
${ }^{1}$ Véase: http://goo.gl/nKnd3p
} 
2. Anticipación de estructuras lingüísticas. Es lo que en nuestro estudio se indica como "aprendizaje de automatismos"

3. Síntesis (véase lo dicho más arriba).

4. Paráfrasis o aproximación: empleo de términos o expresiones sinónimas cuando no se halla el equivalente directo. Esta solución, que también se indica por parte de los intérpretes participantes en nuestro estudio, es especialmente útil para los casos de términos que poseen una referencia sociocultural propia en árabe y carecen de un equivalente directo en español.

5. Alternancia del AME y el árabe informal. Esta solución es especialmente llamativa dado que rompe con la regla no escrita de que el intérprete simultáneo debe siempre verter a la lengua árabe estándar. Asimismo, ofrece una socorrida solución para cuando el equivalente se conoce en el dialectal pero se desconoce no se recuerda en la lengua árabe estándar.

6. Literalidad: traducción literal de fragmentos sin considerar su adecuación al contexto. Esta solución alude a cuando el intérprete pierde el hilo argumental o no lo comprende. Hoy en día no se consideraría una solución ortodoxa puesto que acarrea el peligro del falso sentido cuando no se esté entendiendo el discurso original.

7. Oraciones incompletas: no acabar la frase o no completar la idea. Esta opción también la mencionan los intérpretes de nuestro estudio en forma de omisión. No obstante, entendemos que hay una diferencia entre la "omisión estratégica" (ver guión 8 justo abajo), aquella que se comete a sabiendas para aligerar la reformulación, evitar la redundancia innecesaria o para no incurrir en el falso sentido... y que prácticamente no afecta al sentido original; y la omisión propiamente dicha de unidades de sentido, que se debe evitar a toda costa. Se prefiere siempre dejarse guiar por el contexto para cerrar una idea o volver a reformularla como una unidad de sentido acabada al hecho de dejarla incompleta.

8. Omisiones: silencios y pausas en las que, por su dificultad, no se interpretan determinados fragmentos. Estas serían las "omisiones estratégicas" antes mencionadas.

\section{Enfoques docentes específicos}

La percepción de los encuestados que han recibido o imparten formación en interpretación, y que al mismo tiempo trabajan como intérpretes de conferencias, apoya sin el menor lugar a dudas que las actuaciones formativas consideren las especificidades de esta combinación lingüística. En definitiva, sus respuestas se suman a las conclusiones de toda la literatura especializada, entre otros, de Haddad (2006), quien reclama la adaptación de los ejercicios empleados en la Universidad de Ottawa (Canadá) en los programas de postgrado en interpretación de las universidades sirias; de Hassan (2009), quien defiende la adaptación de la formación para la combinación AR-FR, y Feria (2014), Mahyub Rayaa (2015), quienes reclaman desde diferentes puntos de vista la adopción de un enfoque específico para la enseñanza-aprendizaje de la traducción y la interpretación de AR-ES que tenga en cuenta la realidad lingüística, sociocultural y profesional que la rodea.

\section{Conclusiones e investigación futura}

El objetivo de esta investigación era averiguar qué influencia tiene la asimetría de la combinación lingüística árabe-español en el proceso cognitivo de la IS y cómo se manifiesta en caso de existir tal influencia. En este sentido, las principales conclusiones del estudio son: 
- La muestra de intérpretes encuestada es muy representativa de la realidad profesional en la IS AR-ES y estuvo compuesta por profesionales de muy dilatada experiencia, algunos con experiencia también en la formación de intérpretes.

- Los encuestados afirman rotundamente que la IS AR-ES presenta especificidades debidas a diferentes planos de asimetría, y que ello tiene una incidencia notable en el ejercicio profesional.

- Los encuestados perciben que la asimetría en la combinación AR-ES abarca los planos lingüístico y cultural, que ambos planos se entremezclan, y que esa asimetría es más acusada en la combinación AR-ES que en las combinaciones ES-IN y ES-FR que lo mismos intérpretes ejercen.

- Los encuestados destacan las siguientes especificidades: distancia lingüística y sociocultural, alternancia de código (diglosia o pluriglosia árabe, y alternancia entre árabe e inglés y/o francés), variación (diatópica y diastrática, en particular, variación terminológica) e intertextualidad en lengua árabe (pasajes coránicos y citas poéticas).

- Las impresiones de los encuestados, además de creíbles por su amplia experiencia profesional y docente, coinciden plenamente con toda la literatura previa para las combinaciones AR-IN y AR-FR.

- Los encuestados con formación y/o experiencia docente en interpretación coinciden en que, frente a las tesis defendidas por la Escuela del Sentido, esas especificidades deben tenerse en cuenta durante la formación, que debe adecuarse a cada combinación lingüística.

- Habida cuenta de la trascendencia de la variación en árabe como dificultad específica de la combinación, es preciso instruir al alumnado de IS AR-ES utilizando discursos espontáneos que lo familiaricen con los diferentes acentos y registros árabes. Los encuestados también señalan la importancia de otras estrategias formativas específicas que están en consonancia con lo apuntado en la literatura especializada.

Todo ello afirma varias perspectivas futuras de investigación empírica, entre otras, las siguientes:

- Medir el impacto de la distancia lingüística árabe-español en interpretaciones simultáneas reales. Entre otras cosas, como afirman los encuestados y la literatura especializada, considerando el orden de las palabras y de los sintagmas, y las diferencias discursivas.

- Medir el índice real de variación y alternancia de códigos en oradores árabes en contextos formales reales interpretados por la técnica simultánea y su posible impacto sobre la propia interpretación.

- Investigar si esas asimetrías coincidentes entre español, francés e inglés, por un lado, y árabe, por otro, podrían generalizarse a otras combinaciones que incluyan árabe y otras lenguas indoeuropeas no incluidas en el ámbito cultural del mundo islámico.

A modo de cierre, una recomendación para la formación de futuros intérpretes: en la combinación lingüística árabe-español, en tanto que combinación asimétrica, es de especial interés reforzar la técnica de la anticipación sintáctica y semántica a partir de pistas contextuales, extralingüísticas y unidades fraseológicas. Cuanto mayor sea la competencia lingüística del intérprete, mayor será su capacidad de anticipación de las estructuras del discurso. Asimismo, es necesario potenciar el conocimiento del tipo de texto o situación comunicativa (tipo congresual) para realizar la anticipación extralingüística relativa al perfil de los oradores, las necesidades de los receptores, sus intereses y su postura respecto al tema. Esta fase se adquiere durante el proceso de documentación (Wilss, 1978: 343 y Gile, 1995: 178). 


\section{Referencias}

Aamami, M. (1997). Asmaak al-mutawasit. Trípoli: Dar Al-Jamahiriyah li an-nashr wa attawzi' wa al-'i'lan.

Abdul Aziz, M. H. (2003). “Al-lugha al-'ilmiyah fi al-’asr al-'abbasi”. Al-Logha 4, 9-28.

Abulghar, M. (2003). "Al-lugha wa at-ta'bir al-'ilmi: wijhat nadhar khassah bi-at-ta'lim attibbi", Al-Logha 4, 46-53.

Al-Salman, S. y Al-Khanji, R. (2002). "The Native Language Factor in Simultaneous Interpretation in an Arabic/English Context". Meta 47:4, 607-626.

Badawi, S. (1973). Mustawayat al-arabiyya I-mu'assira fi misr. El Cairo: dar al-ma'arif.

Chiswick, B. R. y Paul W. Miller (2005). "Linguistic Distance: A Quantitative Measure of the Distance between English and Other Languages". Journal of Multilingual and Multicultural Development, 26:1, 1-11.

Bassiouney, R. (2006). Functions of Code Switching in Egypt: Evidence from Monologue. Boston: Brill.

Beenstock, M., et al. (2001). The Effect of Linguistic Distance and Country of Origin on Immigrant Language Skills: Application to Israel. International Migration, 39: 3360.

Borin, L. y Saxena, A. (eds.) (2013). Approaches to Measuring Linguistic Differences. Berlín / Boston: De Gruyter Mouton.

Corriente, F. (1977). A grammatical sketch of the Spanish Arabic dialect bundle. Madrid: Instituto Hispano-Árabe de Cultura.

Crozet, P. (2003). "L'Arabe, langue scientifique: un aperçu historique jusqu'au XIX siècle". Al-Logha 4, 9-27.

Déjean Le Féal, K. (1998). “Non nova, sed nove”. The Intepreters' Newsletter 8, 41-49.

Ferguson, Ch. (1959). “Diglosia”. Word 15, 325-340

Feria, M. C. (2001). La traducción fehaciente del árabe. Fundamentos históricos, jurídicos y metodológicos. Tesis doctoral. Málaga: Universidad de Málaga.

Feria, M. C. (1999). "El traductor-intérprete en la Administración de Justicia". En M. C. Feria (ed.). Traducir para la justicia, 87-108. Granada: Comares.

Ferrando, I. (2001). Introducción a la historia de la lengua árabe: nuevas perspectivas. Zaragoza: Universidad de Zaragoza

Gile, D. (1995). Basic Concepts and Modes for Interpreter and Translator Training. Ámsterdam: John Benjamins. 
Haddad, S. (2006). Interpreting: Confidence-Building Strategies. Damasco: Damascus University Publications.

Hassan, F. (2009). Le débat sur la spécificité de l'interprétation par langues et l'interprétation arabe-français. Tesis doctoral. Lyon: Université Lumière Lyon 2.

Hatim, B. (1997). Communication across Cultures: Translation Theory and Contrastive Text Linguistics. Exeter: University of Exeter Press.

Herrero, B. (1994). "La interpretación en los juzgados". En R. Martin Gaitero (ed.), V encuentros complutenses e tono a la traducción. Instituto Universitario de Lenguas Modernas y Traductores, 687-692. Madrid: Editorial Complutense de Madrid.

Holes, C. (1993). "The use of variation: a study of the political speeches of Gamal Abd EnNasir". Perspectives on Arabic Linguistics 5, 13-45.

Iglesias Fernández, E. (2003). La Interpretación en la Universidad española: estudio empírico de la situación académica y didáctica. Tesis doctoral. Granada: Universidad de Granada.

Ilg, G. (1978). "L'apprentissage de l'interprétation simultanée. De l'allemand vers le français". Paralèles 1, Cahier de l'ETI. University of Geneva.

Kelly, D. et al. (eds.) (2003). La direccionalidad en traducción e interpretación: perspectivas teóricas, profesionales y didácticas. Granada: Atrio

Lamrani, F. (2002). "Arabic Triglossia in the Moroccan criminal courtroom". En A. Youssi et al. (eds.). Aspects of the Dialects of Arabic Today, 299-306. Rabat: Amapatril.

Longley, P. (1978). "A integrated programme for training interpreters". En D. Gerver and H.W. Sinaiko (eds.). Language interpretation and communication, 45-56. Nueva York: Plenum Press.

Mahyub Rayaa, B. (2020). "Arabic-Spanish simultaneous interpreting: training and professional practice", 350-365. En H. El Farahaty et al. The Routledge Handbook of Arabic Translation. Londres: Routledge.

Mahyub Rayaa, B. (2015). La interpretación simultánea árabe-español y sus peculiaridades. Docencia y profesión. Tesis doctoral. Granada: Universidad de Granada.

Mahyub Rayaa, B. (2013). "La interpretación simultánea árabe-español y sus peculiaridades: docencia y profesión. Estudio piloto. En O. Becerra et al. Quality in interpreting: widening the scope, 337-365. Granada: Comares.

Mahyub Rayaa, B. y Zarrouk, M. (2013). Interpretación Simultánea (Árabe-Español). método para la enseñanza-aprendizaje. Toledo: Escuela de Traductores de Toledo, Universidad de Castilla-La Mancha.

Manuel Jerez, J. (2006). La incorporación de la realidad profesional a la formación de intérpretes de conferencias mediante las nuevas tecnologías y la investigaciónacción. Tesis doctoral. Granada: Universidad de Granada. 
Moshref, O. (2012). Approaches to Diglossic Mixing in Spoken Arabic: Review of some corpus studies. Paper presented at Illinois Symposium on Semitic Linguistics, University of Illinois, Urbana-Champaign, IL.

Ortega Herráez, J. M. (2007). "La interpretación árabe-español en los juzgados y tribunales penales españoles: el intérprete y su papel profesional". Puentes 8,11 24.

Ortega Herráez, J. M. (2010). Interpretar para la Justicia. Granada: Comares.

Seleskovitch, D. y Lederer, M. (1984). Interpréter pour traduire. París: Publications de la Sorbonne: Didier Érudition.

Stenzl, C. (1989). "From theory to practice and from practice to theory". In L. Gran and J. Dodds (eds.). Theoretical and Practical Aspects of Teaching Conference Interpretation (pp. 23-26). Udine: Campanotto.

Taibi, M. (2007). "El árabe en el ámbito de la traducción e interpretación en los Servicios Públicos". Puentes 8, 5-10.

Versteegh, K. (1997). The Arabic Language. Edimburgo: Edinburgh University Press.

Wilmsen, D. (2003). "One global standard or multiple regional standards? A problem in the practice and pedagogy of Arabic interpreting". En A. Collados Aís, et al. (eds.). La evaluación de la calidad de la interpretación: docencia y profesión, 69-79. Granada: Comares.

Wilmsen, D. y Osama Youssef, R. (2009). "Regional standards and local routes in adoption techniques for specialised terminologies in the dialects of written Arabic". The Journal of Specialised Translation 11, 191-210.

Wilss, W. (1978). "Syntactic anticipation in German-English". En D. Gerver and H.W. Sinaiko (eds.). Language interpretation and communication, 343-352. Nueva York: Plenum Press.

Youssi, A. (1983). "La triglossie dans la typologie linguistique”. La Linguistique 19: 2, 71 83. 